\title{
On the Adequacy of Principal Factor Analysis for the Study of Shape Variability
}

\author{
Miguel Ángel González Ballester ${ }^{a, b}$, Marius George Linguraru ${ }^{b, c}$, \\ Mauricio Reyes Aguirre ${ }^{b}$ and Nicholas Ayache ${ }^{b}$ \\ ${ }^{a}$ University of Bern, MEM Research Center, \\ Institute for Surgical Technology and Biomechanics, \\ Stauffacherstrasse 78, CH-3014 Bern, Switzerland \\ http://memcenter unibe.ch \\ ${ }^{b}$ INRIA, Epidaure Research Project, \\ 2004 route des lucioles BP 93, Sophia Antipolis, 06902 France \\ http://www.inria.fr/epidaure \\ ${ }^{c}$ Harvard University, Biorobotics Laboratory, \\ 29 Oxford Street, Cambridge MA 02138, USA \\ http://biorobotics.harvard.edu
}

\begin{abstract}
The analysis of shape variability of anatomical structures is of key importance in a number of clinical disciplines, as abnormality in shape can be related to certain diseases. Statistical shape analysis techniques commonly employed in the medical imaging community, such as Active Shape Models or Active Appearance Models rely on Principal Component Analysis (PCA) to decompose shape variability into a reduced set of interpretable components. In this paper we propose Principal Factor Analysis (PFA) as an alternative to PCA and argue that PFA is a better suited technique for medical imaging applications. PFA provides a decomposition into modes of variation that are more easily interpretable, while still being a linear, efficient technique that performs dimensionality reduction (as opposed to Independent Component Analysis, ICA). Both PCA and PFA are described. Examples are provided for 2D landmark data of corpora callosa outlines, as well as vector-valued 3D deformation fields resulting from non-rigid registration of ventricles in MRI. The results show that PFA is a more descriptive tool for shape analysis, at a small cost in size (as in theory more components may be necessary to explain a given percentage of total variance in the data). In conclusion, we argue that it is important to study the potential of factor analysis techniques other than PCA for the application of shape analysis, and defend PFA as a good alternative.
\end{abstract}

Keywords: Shape, statistical models, principal factor analysis, principal component analysis, morphometry, registration

\section{INTRODUCTION}

The analysis of shape variability of anatomical structures is of key importance in a number of clinical disciplines, as abnormality in shape can be related to certain diseases. Examples in neurology include the study of brain asymmetry to verify its relation to schizophrenia, ${ }^{9}$ or the detection and quantification of atrophy as a correlate to multiple sclerosis. ${ }^{1}$

Statistical shape analysis techniques enjoy a remarkable popularity within the medical image analysis community. Its flagship, the Active Shape Model (ASM), proposed by Cootes et al., ${ }^{5}$ provides a method to study the structure of point data sets or meshes. ${ }^{8}$ This technique was later extended to intensity information, and thus image data, as the Active Appearance Model (AAM). ${ }^{6}$

Address for correspondence: Miguel.Gonzalez@MEMcenter.unibe.ch 
Nearly all existing statistical shape analysis methods rely on Principal Component Analysis (PCA) to build a compact model of principal "modes of variation" from a training set. PCA belongs to a family of methods for multivariate analysis commonly known as Factor Analysis (FA). Reviews and comparative studies of FA techniques can be found elsewhere. ${ }^{3,7,16}$ Such techniques can be classified into linear and non-linear, reflecting whether the shape variation can be expressed as a linear combination of basic deformation primitives. We contend that a factorial decomposition of shape variability, if it is to be easily interpretable, must follow a linear model, where each mode of variation has a scalar weight. PCA is a linear technique. Exceptionally, another linear FA method, Independent Component Analysis (ICA),${ }^{17}$ or equivalently Maximum Autocorrelation Function (MAF) PCA, ${ }^{10,13}$ has been proposed. However, ICA does not provide a compact representation of shape variability, as one obtains as many independent components as there are variables. ${ }^{7,17}$

A common difficulty encountered when using PCA for shape analysis is that of correlating the resulting modes of variation with intuitive shape descriptions employed by clinical partners. Thus, these components are often described as combinations of several localised shears, twists, rotations, etc., but these are most often simplistic approximations to complex deformation fields.

In this paper we compare the performance of PCA with another linear factor analysis technique, known as Principal Factor Analysis (PFA). According to the FA literature, PCA is recommended for dimensionality reduction, whereas PFA is adapted for the study of structure in the data. In particular, Widaman ${ }^{18}$ states that $^{2}$ "principal component analysis should not be used if a researcher wishes to obtain parameters reflecting latent constructs or factors." This paper will prove that PFA provides a better decomposition of shape variation in terms of interpretability.

Sections 2 and 3 describe PCA and PFA, respectively. Section 4 shows results of both techniques on 2D landmark data sets, and section 5 provides results for the analysis of 3D vector fields. Discussion and conclusions are provided in section 6 .

\section{PRINCIPAL COMPONENT ANALYSIS}

PCA is a projection model for FA, aiming at finding a low-dimensional manifold in the space of the data, such that the distance between the data and its projection on the manifold is small. ${ }^{16}$ PCA is the best, in the mean-square error sense, linear dimension reduction technique. ${ }^{7}$

Given a set of training data $\left\{\vec{t}_{1}, \vec{t}_{2}, \ldots, \vec{t}_{N}\right\}$ in a given orthonormal basis of $\Re^{D}$, PCA finds a new orthonormal basis $\left\{\vec{u}_{1}, \ldots, \vec{u}_{D}\right\}$ with its axes ordered. This new basis is rotated such that the first axis is oriented along the direction in which the data has its highest variance. The second axis is oriented along the direction of maximal variance in the data, orthogonal to the first axis. Similarly, subsequent axes are oriented so as to account for as much as possible of the variance in the data, subject to the constraint that they must be orthogonal to the preceding axes. Consequently, these axes have associated decreasing "indices" $\lambda_{d}, d=1, \ldots, D$, corresponding to the variance of the data set when projected on the axes. The principal components are the set of new ordered basis vectors.

The way to find the principal components is to compute the sample covariance matrix of the data set, $\vec{S}$, and then find its eigen-structure

$$
\overrightarrow{S U}=\overrightarrow{U \Lambda}
$$

$\vec{U}$ is a $D \times D$ matrix which has the unit length eigenvectors $\vec{u}_{1}, \ldots, \vec{u}_{D}$ as its columns, and $\vec{\Lambda}$ is a diagonal matrix with the corresponding eigenvalues $\lambda_{1}, \ldots, \lambda_{D}$. The eigenvectors are the principal components and the eigenvalues their corresponding projected variances. ${ }^{16}$

\section{PRINCIPAL FACTOR ANALYSIS}

In opposition to PCA, which is a projection model, PFA can be considered as a generative model for FA. Generative models try to model the density function that is assumed to have generated the data, under a set of constraints that restricts the set of possible models to those with a low intrinsic dimensionality. ${ }^{16}$ The following description is mainly based on Carreira-Perpiñán's paper. ${ }^{3}$ 
PFA represents an observed $D$-dimensional continuous variable, $\vec{t}$, as a linear function $\vec{f}$ of an $L$-dimensional $(L<D)$ continuous latent variable $\vec{x}$ and an independent Gaussian noise process:

$$
\vec{t}=\overrightarrow{\Lambda x}+\vec{\mu}+\vec{e}
$$

Here $\vec{\Lambda}$ is the $D \times L$ factor loading matrix defining the linear function $\vec{f}, \vec{\mu}$ is a $D$-dimensional vector representing the mean of the distribution of $\vec{t}$, and $\vec{e}$ is a $D$-dimensional vector representing the noise or individual variability associated with each of the $D$ observed variables.

PFA assumes a Gaussian distributed prior and noise model, and a linear mapping from data space to latent space. Specifically:

- The latent space prior $p(\vec{x})$ is unit normal:

$$
p(\vec{x}) \sim N(\overrightarrow{0}, \vec{I})
$$

The latent variables $\vec{x}$ are often referred to as factors.

- The smooth mapping $\vec{f}$ is linear:

$$
\vec{f}(\vec{x})=\overrightarrow{\Lambda x}+\vec{\mu}
$$

The columns of the $D \times L$ matrix $\vec{\Lambda}$ are referred to as factor loadings.

- The data space noise model $\vec{e}$ is normal centered in $\vec{f}(\vec{x})$ with diagonal covariance matrix $\vec{\Psi}$ :

$$
p(\vec{t} \mid \vec{x}) \sim N(\vec{f}(\vec{x}), \vec{\Psi})
$$

The $D$ diagonal elements of $\vec{\Psi}$ are referred to as the uniqueness.

The marginal distribution in data space is normal with a constrained covariance matrix:

$$
p(\vec{t} \mid \vec{x}) \sim N\left(\vec{\mu}, \overrightarrow{\Lambda \Lambda}^{t}+\vec{\Psi}\right)
$$

Following Bayes' rule, the posterior in latent space is also normal:

$$
\begin{gathered}
p(\vec{x} \mid \vec{t}) N\left(\vec{A}(\vec{t}-\vec{\mu}),\left(\vec{I}+\vec{\Lambda}^{t} \vec{\Psi}^{-1} \vec{\Lambda}\right)^{-1}\right) \\
\vec{A}=\vec{\Lambda}^{t}\left(\overrightarrow{\Lambda \Lambda}^{t}+\vec{\Psi}\right)^{-1}=\left(\vec{I}+\vec{\Lambda}^{t} \vec{\Psi}^{-1} \vec{\Lambda}\right)^{-1} \vec{\Lambda}^{t} \vec{\Psi}^{-1}
\end{gathered}
$$

Note that we can define the analog of the inverse of $\vec{f}$ by taking the posterior mean as a reduced-dimension representative of $\vec{t}$ :

$$
\vec{F}(\vec{t})=E\{\vec{x} \mid \vec{t}\}=\vec{A}(\vec{t}-\vec{\mu})
$$

The inverse mapping $\vec{F}$ is a smooth function mapping each data point to a point in latent space.

The parameters of the PFA model may be estimated using the EM (Expectation-Maximization) algorithm ${ }^{15}$ :

- E step: This requires computing the moments. For each data point $\vec{t}_{n}$ given the current parameter values $\vec{\Lambda}(\tau)$ and $\vec{\Psi}^{(\tau)}$ :

$$
\begin{gathered}
E\left\{\vec{x} \mid \vec{t}_{n}\right\}=\vec{A}^{(\tau)}\left(\vec{t}_{n}-\vec{\mu}\right) \\
E\left\{\overrightarrow{x x}^{t} \mid \vec{t}_{n}\right\}=\vec{I}-\vec{A}^{(\tau)} \vec{\Lambda}^{(\tau)}+\vec{A}^{(\tau)}\left(\vec{t}_{n}-\vec{\mu}\right)\left(\vec{t}_{n}-\vec{\mu}\right)^{t}\left(\vec{A}^{(\tau)}\right)^{t}
\end{gathered}
$$


- M step: This results in the following update equations for the factor loadings $\vec{\Lambda}$ and uniqueness $\vec{\Psi}$ :

$$
\begin{aligned}
& \Lambda^{(\overrightarrow{\tau+1})}=\left(\sum_{n=1}^{N} \vec{t}_{n} E\left\{\vec{x} \mid \vec{t}_{n}\right\}^{t}\right)\left(\sum_{n=1}^{N} E\left\{\overrightarrow{x x}^{t} \mid \vec{t}_{n}\right\}^{t}\right)^{-1} \\
& \Psi^{(\overrightarrow{\tau+1})}=\frac{1}{N} \operatorname{diag}\left(\sum_{n=1}^{N} \vec{t}_{n} \vec{t}_{n}^{t}-\vec{\Lambda}^{(\tau+1)} E\left\{\vec{x} \mid \vec{t}_{n}\right\} \vec{t}_{n}^{t}\right)
\end{aligned}
$$

where the updated moments are used and the "diag" operator sets all off-diagonal elements of a matrix to zero. The location parameter $\vec{\mu}$ is estimated by the sample mean, and does not take part in the EM algorithm

Note that as opposed to PCA, the factor decomposition is not unique, since an orthogonal rotation of the factors $\left(\vec{\Lambda}^{\prime}=\overrightarrow{\Lambda R}\right.$ where $\vec{R}$ is an orthogonal matrix) does not alter the distribution in data space, $p(\vec{t})$. Thus, from all the factor loading matrices $\vec{\Lambda}$, we are free to choose that which is easiest to interpret according to some criterion. We employ Varimax rotation, ${ }^{12}$ which finds an orthogonal rotation of the factors such that, for each new factor, the loadings are either very large or very small (in absolute value). The resulting rotated matrix $\vec{\Lambda}^{\prime}$ has many values clamped to (almost) 0 , that is, each factor involves only a few orthogonal variables. This simplifies factor interpretation.

\section{RESULTS ON 2D LANDMARK DATA}

In this first experiment we attempt to compare the performance of PFA vs. PCA in the analysis of the shape variability of the corpus callosum. Our training data consists of 9 sets of 2D landmarks delimiting the corpora callosa of 9 patients as evidenced on MRI. Each data set has 63 landmarks. The delineations were performed manually by an expert. These data sets were kindly provided by Prof. Anand Rangarajan, from the Dept. of Computer and Information Science and Engineering of the University of Florida.

The average shape was computed by simple averaging of corresponding landmarks across the data sets. The remaining variation was analysed by PCA and then by PFA with Varimax rotation of the resulting factors.

Figure 1 shows the 3 principal components (top) and 3 principal factors (bottom) resulting from PCA and PFA, respectively. PFA creates factors that are more easily interpretable and correspond to intuitive concepts. PCA does a good job at modeling the variance of the data in a few principal modes of variation, but such modes can be interpreted as a combination of several basic displacements, which can be rather complex in many cases. On the contrary, PFA decomposes the variation into simple factors that have one or two defined directions of displacement.

\section{RESULTS ON VECTOR-VALUED DISPLACEMENT FIELDS}

The following example considers the case of the analysis of 3D vector-valued displacement fields resulting from non-rigid registration. In particular, 6 brain MRI data sets where registered to the Brainweb atlas of the Montreal Neurological Institute ${ }^{4}$ for spatial normalization. To this end, we employed a block-matching registration algorithm developed in our laboratory ${ }^{14}$ to find the best affine transformation that matched each data set to the atlas.

In order to avoid bias from using one of the data sets as a reference for non-rigid registration, we opted for using a standard segmented data set, known as the Zubal Phantom, ${ }^{19}$ which we previously registered affinely to the Brainweb phantom as for the previous data sets (see Fig. 2).

The aim of this study was to determine predominant patterns in ventricular shape variability. A mask containing the ventricles was extracted from each MR image (by thresholding and mathematical morphology) and from the Zubal Phantom (simply selecting the appropriate labels from the segmented image). The PASHA algorithm $^{2}$ was employed for non-rigid registration of the Zubal phantom mask to the ventricle mask of each 

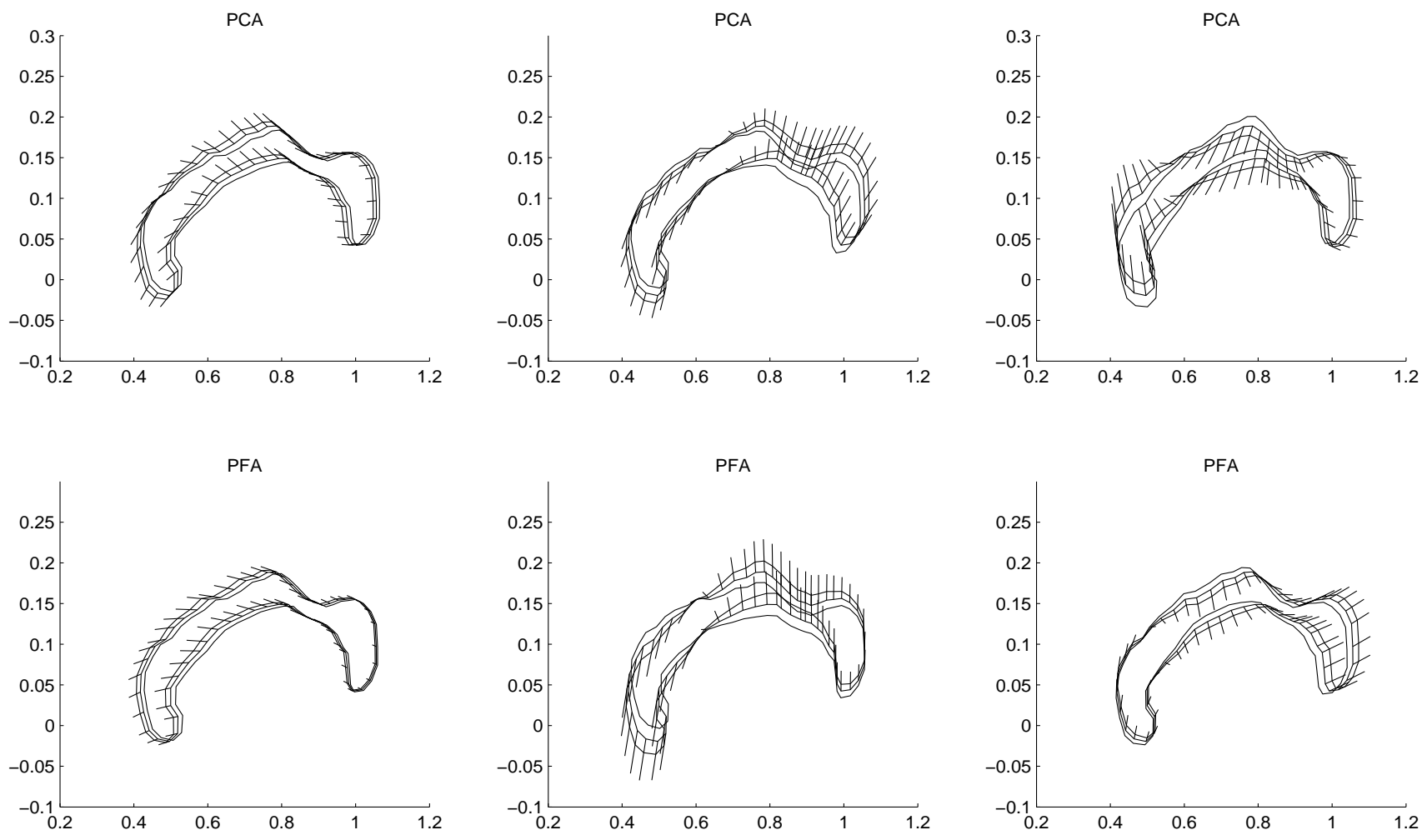

Figure 1. PCA and PFA results on 2D landmark data sets representing corpora callosa. Top: First 3 principal components (ordered from left to right according to the variance explained), and bottom: first 3 principal factors after Varimax rotation. PFA provides modes of variation that are more easily interpretable and intuitive.

patient (Fig. 3). This results in a set of 3D deformation fields describing the displacement of each point in the Zubal phantom to match each of the patient MRI data sets (Fig. 4).

The subsequent shape analysis focuses on the displacement vectors associated to the ventricular boundary. A mask containing the voxels in the outer boundary of the ventricles is extracted from the Zubal phantom mask by simple mathematical morphology. This mask is used to select the corresponding sites in the vector fields for each patient (see Fig. 4).

We can now proceed to analyze the structure of the vector field, by computing a mean displacement field and extracting a reduced set of principal modes of variation around such mean displacement pattern. PCA and PFA were performed on the set of 6 ventricle displacement fields.

For comparison, figure 5 shows the vector norm of the first principal component (left) and principal factor (right). It can be observed that the effect of the principal factor is more localized (towards the back of the ventricles), while the principal component affects most of the ventricular surface.

\section{DISCUSSION AND CONCLUSIONS}

The key difference between PFA and PCA is that PFA models covariance between variables, rather than the total variance in the data. PCA determines the factors which account for the total (unique and common) variance in the set of variables; PFA determines the least number of factors which can account for the common variance (correlation).

PFA provides better interpretability than PCA, as evidenced by the results provided. The superiority of PFA over PCA has also been highlighted recently for fMRI analysis. ${ }^{11}$ On the other hand, PCA is optimal in 

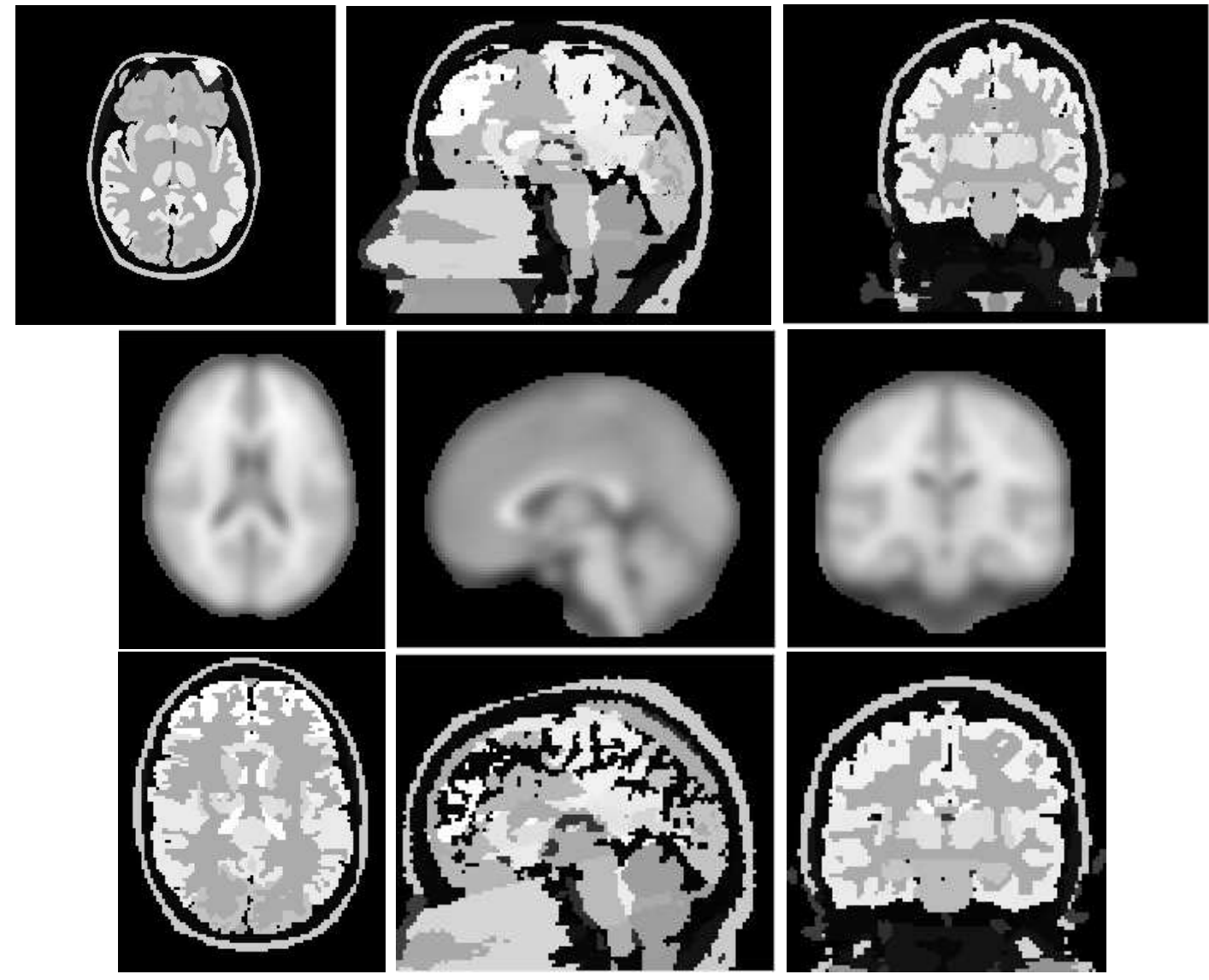

Figure 2. The Zubal phantom (top) contains a colour-coded labelling of anatomical structures. This image, as well as the patient images, is registered to the MNI phantom (middle) via an affine transformation. The results of this registration on the Zubal phantom are shown (bottom).
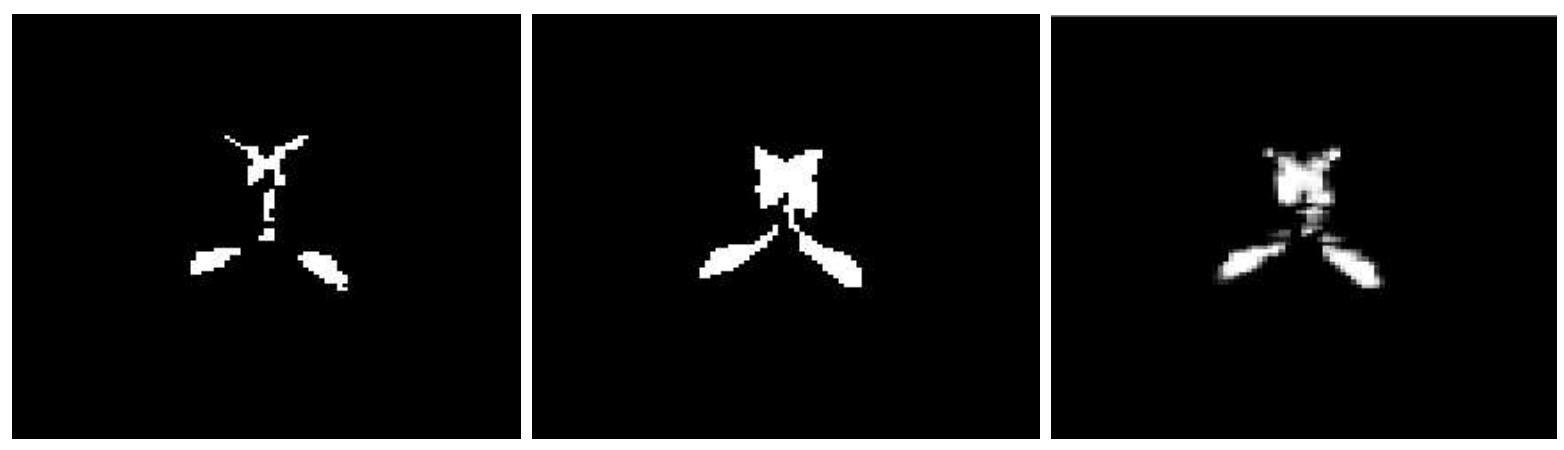

Figure 3. The mask corresponding to the ventricles on the Zubal phantom (left) was extracted simply by isolating the corresponding intensity value. Similary, the ventricle mask for the patient image (middle) was extracted by thresholding and morphological operations. Finally, the Zubal mask was warped to the shape of the patient ventricle (right). 

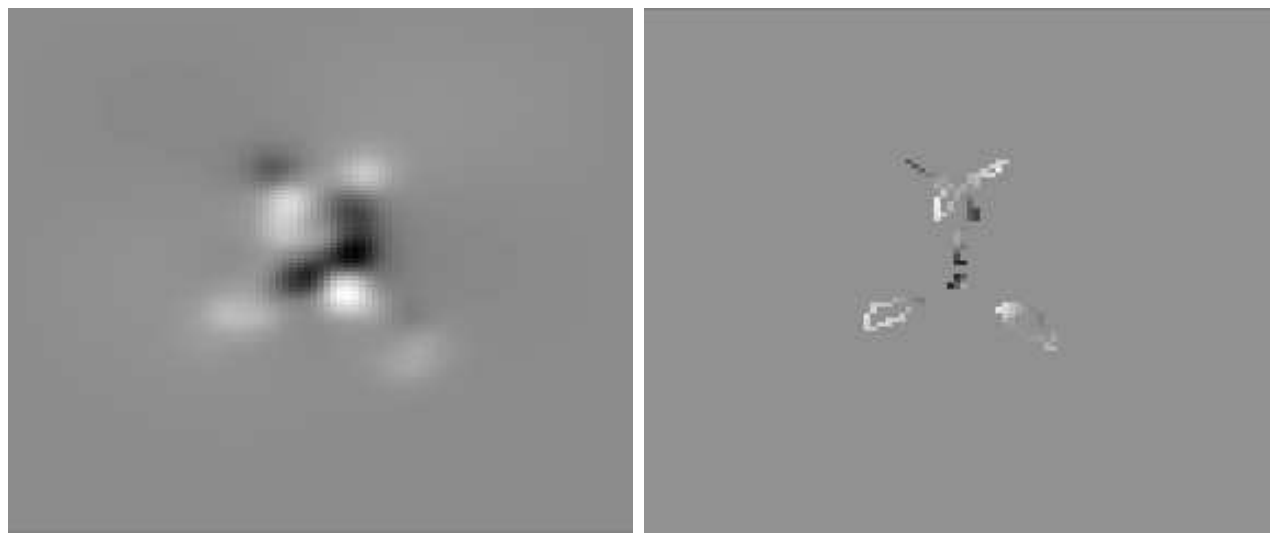

Figure 4. Deformation field (only x component is shown) mapping the Zubal ventricle mask to the ventricle mask of one of the patients. On the right, the voxels corresponding to the outer boundary of the ventricles were isolated.
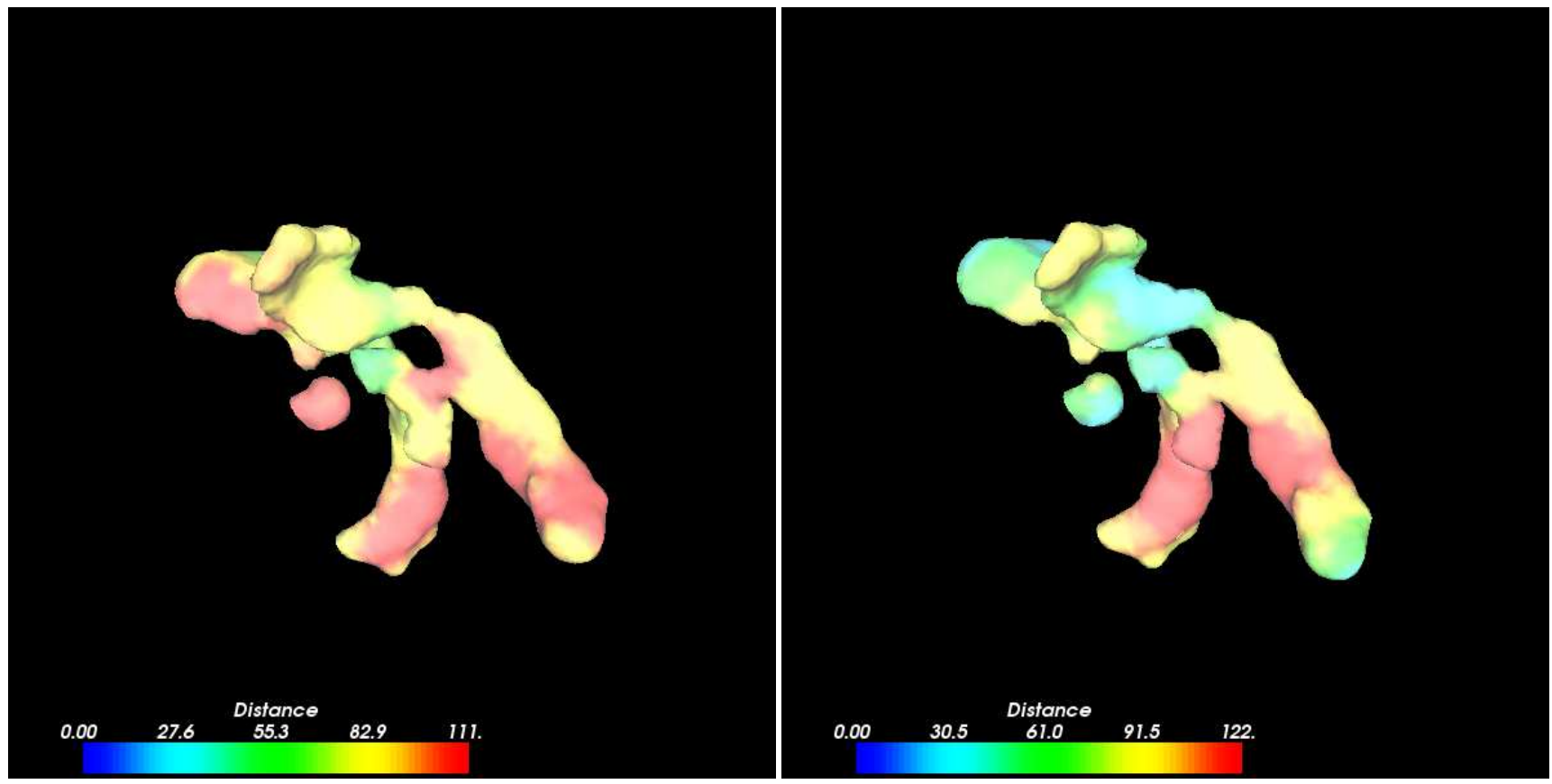

Figure 5. 3D view of the norm of the first principal component (left) and principal factor (right) extracted from a training set of $3 \mathrm{D}$ displacement vector fields on the surface of brain ventricles. PFA factors have a more localized effect (in the back of the ventricles). 
reducing dimensionality, so PFA decomposition may require more factors to reconstruct the original data up to a certain threshold of accuracy.

Alternative factor analysis techniques may be employed. ${ }^{7}$ An exhaustive comparative study of such techniques for statistical shape analysis is in progress.

\section{Acknowledgments}

We would like to thank Prof. Anand Rangarajan, from the Dept. of Computer and Information Science and Engineering of the University of Florida, for kindly providing the 2D landmark data sets employed in section 4.

\section{REFERENCES}

1. Bakshi, R., Benedict, R. H., Bermel, R. A., Jacobs, L.: Regional Brain Atrophy is Associated with Physical Disability in Multiple Sclerosis: Semiquantitative MRI and Relationship to Clinical Findings. Journal of NeuroImaging, 11(2):129-136, 2001

2. Cachier, P., Bardinet, E., Dormont, D., Pennec, X., Ayache, N.: Iconic Feature Nonrigid Registration: the PASHA Algorithm. Computer Vision and Image Understanding, 24(4-5):801-813, 2003

3. Carreira-Perpiñán, M. A., Renals, S.: Dimensionality Reduction of Electropalatographic Data Using Latent Variable Models. Speech Communication, 26(4):259-282, 1998

4. Collins, D.L. et al.: Design and Construction of a Realistic Digital Brain Phantom. IEEE Transactions on Medical Imaging, 17(3):463-468, 1998

5. Cootes, T. F., Taylor, C. J., Cooper, D. H., Graham, J.: Active Shape Models - Their Training and Applications. Computer Vision and Image Understanding, 61(2), 1995

6. Cootes, T. F., Edwards, G. J., Taylor, C. J.: Active Appearance Models. IEEE Transactions on Pattern Analysis and Machine Intelligence, 23(6):681-685, 2001

7. Fodor, I.K.: A Survey of Dimension Reduction Techniques. Lawrence Livermore National Laboratory Technical Report no. UCRL-ID-148494, 2002

8. González Ballester, M. A., Zisserman, A., Brady, M.: Measurement of Brain Structures based on Statistical and Geometrical 3D Segmentation. Procs. of MICCAI'1998, Lecture Notes in Computer Science, 1496:499508,1998

9. González Ballester, M. A., Zisserman, A., Brady, M.: Estimation of the Partial Volume Effect in MRI. Medical Image Analysis, 6(4):389-405, 2002

10. Hilger, K. B., Larsen, R., Wrobel, M. C.: Growth Modeling of Human Mandibules Using Non-Euclidean Metrics. Medical Image Analysis, 7(4):417-423, 2003

11. Huang, C.-C., Liou, M., Cheng, P. E., Chen, C.-C.: Noise Reduction in Functional MR Images by Common Factor Models. Procs. of International Conference on Neural Information Processing (ICONIP), 2001

12. Kaiser, H. F.: The Varimax Criterion for Analytic Rotation in Factor Analysis. Psychometrika, 23(3):187200, 1958

13. Larsen, R., Hilger, K. B.: Statistical Shape Analysis Using Non-Euclidean Metrics. Medical Image Analysis, 7(4):417-423, 2003

14. Ourselin, S., Roche, A., Prima, S., Ayache, N.: Block Matching: a General Framework to Improve Robustness of Rigid Registration of Medical Images. Procs. of MICCAI'2000, Lecture Notes in Computer Science, 1935:557-566, 2000

15. Rubin, D. B., Thayer, D. T.: EM Algorithms for ML Factor Analysis. Psychometrika, 47(1):69-76, 1982

16. Svensén, J. F. M.: GTM: The Generative Topographic Mapping. PhD Thesis, Aston University, 1998

17. Üzümcü, M., Frangi, A. F., Sonka, M., Reiber, J. H. C., Lelieveldt, B. P. F.: ICA vs. PCA Active Appearance Models: Application to Cardiac MR Segmentation. Procs. of MICCAI'2003, Lecture Notes in Computer Science, 2878:451-458, 2003

18. Widaman, K.F.: Common Factor Analysis versus Principal Components Analysis: Differential Bias in Representing Model Parameters? Multivariate Behavioral Research, 28:263-311, 1993

19. Zubal, I. G. et al.: Computerized Three-Dimensional Segmented Human Anatomy. Medical Physics, 21:299302,1994 